processes and mediate evolutionary diversification than the transcription factors themselves. This makes sense because, in most cases, sequence-specific transcription factors or the signalling proteins that modulate their activity are highly conserved among organisms with very different shapes and forms. Yet the constellations of cis-regulatory elements, which together make up the cis-regulatory control units that dictate the time, place and magnitude of gene expression, are more diverse and seem to evolve more rapidly than the transcription factors that recognize them. Furthermore, such control units also dictate the expression patterns of transcription factors and signalling proteins in time and space, and thereby determine the exact patterns and repertoires of developmental gene expression.

In general, Davidson does an excellent job of reducing the complexity of different developmental pathways and modes of embryonic development in diverse animal phyla to a set of simplified and logical concepts and principles. He provides excellent illustrations and experimental examples derived from several model organisms: nematode worms, fruitflies, sea urchins, tunicates and vertebrates of different sorts. What is especially attractive about the book are the regulatory networks drawn as simple wiring and computational diagrams. These go a long way towards explaining the basic regulatory logic and engineering principles of some of the most complex biological phenomena: animal development and the evolution of body forms.

This book should be read by all biologists who want to understand how development and evolution take place and what governs the workings of genomes. I also recommend it to computer scientists and engineers who are interested in the budding field of computational biology, as reading it does not require an extensive background in developmental biology.

Michael Karin is in the Department of Pharmacology, University of California, San Diego School of Medicine, La Jolla, California 92093, USA.

\title{
Putting DNA on the map
}

\section{Reconceiving the Gene: Seymour Benzer's Adventures in Phage Genetics by Frederic Lawrence Holmes \\ Yale University Press: 2006.320 pp. \$50}

\section{Denis Thieffry}

In the 1950s, Seymour Benzer set out on a daunting research programme aimed at resolving the fine structure of the gene. Using bacteriophages and various strains of bacteria, his genetic-mapping enterprise ultimately united genetics and structural chemistry. How did Benzer conceive and achieve this goal? And what where the critical components and influences that led to the success of his enterprise?

In Reconceiving the Gene, Larry Holmes addresses these questions in a scrupulous and captivating historical analysis. Making extensive use of Benzer's laboratory notebooks, scientific correspondence, reports and grant proposals, and complementing these with interviews, Holmes carefully retraces Benzer's sinuous investigative path and offers us a well-documented day-to-day analysis of the development of his work. Although Benzer had previously published his own biographical recollection of this period as a chapter in the edited volume Phage and the Origins of Molecular Biology (Cold Spring Harbor Laboratory Press, 1966), Holmes' extensive analysis shows how Benzer sometimes condensed events in his relatively sketchy autobiographical reconstruction.

Trained as a solid-state physicist, Benzer was well prepared for quantitative analyses and genetic abstraction. In two years of training at the California Institute of Technology and a year at the Pasteur Institute in Paris, Benzer progressively penetrated the informal international phage network. Initially, he was deeply influenced by key players of the phage group, including Max Delbrück, Salvador Luria, André Lwoff, François Jacob, Alfred Hershey and Sidney Brenner. However, he progressively altered his pathway in the light of

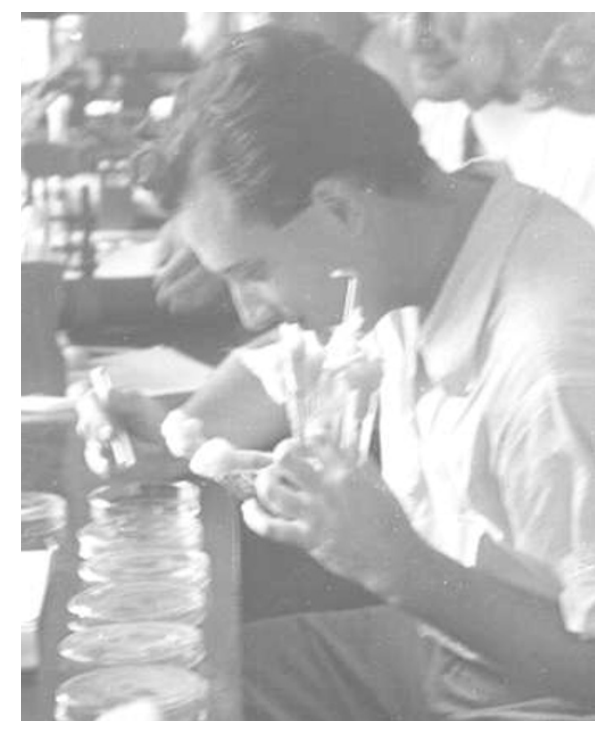

Fine work: Seymour Benzer created a map of the gene with a resolution of just a few nucleotides.

his own results, as well as those of others notably an experiment by Hershey and Martha Chase demonstrating the hereditary role of phage DNA, and James Watson and Francis Crick's work to develop the double-helix model of DNA.

Holmes ends his book with extensive accounts of the main public presentations by Benzer of his mapping results. About Benzer's landmark publication (Proc. Natl Acad. Sci. USA 41, 344-354; 1955), Holmes states: "Like most modern scientific papers, Benzer's 'Fine Structure of a Genetic Region in Bacteriophage' is a logical reconstruction of the experiments, observations, and arguments supporting his conclusions. It has little narrative structure and does not purport to follow the investigative pathway from which it came."

Holmes patiently reconstructs the investigative path that led Benzer to draw the first high-resolution genetic map (down to a few nucleotides). The map supports the contention that hereditary units are ordered linearly on the chromosome, and that genetic mutations and crossing-over experiments could be traced back to the underlying linear arrangement of DNA. Holmes says that Benzer isolated, characterized and crossed about a thousand T4 phage mutants, most of them in the limited rII region. This was possible thanks to the fantastic resolving power of Benzer's experimental system, which he progressively improved by using different types of mutants (extended deletions) to speed the mapping of novel mutants.

Benzer's results reached a wide audience through his contributions to two major conferences: the Brookhaven Symposium on Biology in 1955 and the McCollum-Pratt Institute Symposium on the Chemical Basis of Heredity at Johns Hopkins University in 1956. On the latter occasion, Benzer proposed three new terms - cistron, recon and muton - to solve the ambiguities associated with the term 'gene', which is considered altogether as a unit of function, recombination (crossing-over) and mutation. Although these terms (perhaps with the exception of 'cistron') did not become really popular among the emerging molecularbiology community, these distinctions helped to clarify the relationships between the different gene definitions. For a broader historical analysis, see The Concept of the Gene in Development and Evolution, edited by Peter Beurton, Raphael Falk and Hans-Jörg Rheinberger (Cambridge University Press, 2000).

Although technical in places, Reconceiving the Gene should nevertheless be accessible to a wide scientifically literate audience as it further introduces the broader context of classical and phage genetics. Holmes initially planned to go beyond Benzer's fine rII mapping and also cover his contributions towards solving the genetic code. This plan was impeded by illness, however, and Holmes died on $27 \mathrm{March}$ 2003. Hopefully, his captivating book will stimulate other historians of biology to complete his project.

Denis Thieffry is in the Faculté des Sciences de Luminy, Université de la Méditerranée,

13288 Marseille, France. 